\title{
An Integrated Analysis of Aberrantly Expressed miRNA and mRNA Profiles Unveils a Robust Regulatory Network in HepG2 Cell ${ }^{*}$
}

\author{
Sheng Yang, Hui Zhang, Li Guo, Yang Zhao, Feng Chen ${ }^{\#}$ \\ Department of Epidemiology and Biostatistics, Nanjing Medical University (NJMU), Nanjing, China \\ Email: kyny2011@hotmail.com, zhanghui317@yeah.net, lguo@njmu.edu.cn, \\ zhaoyang@njmu.edu.cn, "Fengchen@njmu.edu.cn \\ Received October 2012
}

\begin{abstract}
As crucial negative regulatory small non-coding molecules, microRNAs (miRNAs), have multiple biological roles. The abnormal expression of specific miRNAs may contribute to the occurrence and development of tumor. Here, based on HepG2 and L02 cells, we attempted to demonstrate the potential regulatory network of aberrantly expressed miRNA profiles, interaction between miRNA and mRNA, and potential functional correlation between different miRNAs. Deregulated miRNA and mRNA expression profiles were completely surveyed and identified by applying deep sequencing and microarray techniques, respectively. The genome-wide and integrative analysis of miRNA-mRNA was performed based on their functional relationship according to experimentally validated and predicted targets. Nearly 50\% targets were negatively regulated by at least 2 aberrantly expressed miRNAs. Similar results were obtained based on experimentally validated and predicted targets. Compared with abnormal miRNAs, their targets showed various expression patterns: stably expressed, down-regulated or up-regulated. Although the theoretical potential miRNA-mRNA interaction could be predicted, they showed consistent or inconsistent expression patterns. Both functional enrichment analysis of target mRNAs of dysregulated miRNAs and abnormal mRNA profiles suggested that corresponding pathways were involved in tumorigenesis. Moreover, to obtain potential functional relationships between different miRNAs, we also performed expression analysis of homologous miRNAs in gene families. Generally, they could co-regulate biological processes with similar roles. The integrative analysis of miRNA-mRNA indicated a complex and flexible regulatory network. The robust network mainly derived from multiple targets for a specific miRNA (and vice versa), each mRNA and co-regulation roles of different miRNAs.
\end{abstract}

Keywords: miRNA (microRNA); mRNA; Intergrated Analysis; Hepatoma Carcinoma Cell

\section{Introduction}

MicroRNAs (miRNA) are not only the most conserved but also special non-coding RNAs which guide RNA silencing. These different characters are given rise to the disparate structure and biogenesis [1]. Mature miRNA with a length of approximately 22 nucleotides (nt) is a single strand and processes from a stem-loop precursor miRNA (pre-miRNA) molecule (60 - 120 nt), with the assistance of DISER. Before the precursor forming, primary miRNA (pri-miRNA) is cleaved by a ribonuclease (RNase), such as DROSHA. After processing the miRNA duplex, one strand, called mature or active miRNA,

\footnotetext{
"The work was supported by the project of 81072389 from National Natural Science Foundation of China and a Project Funded by the Priority Academic Program Development of Jiangsu Higher Education Institutions (PAPD).

${ }^{\#}$ Corresponding author.
}

is loaded into AGO protein to participate the post-transcriptional procedure. Then the single strand miRNA is incorporated with RNA-inducing silencing complex (RISC) that interacts with 3' untranslated region (UTR) of messenger RNAs (mRNAs) through base pairing to facilitate mRNAs repression or degradation [2-4].

Many experimental and bioinformatics analysis evidences indicate that one single miRNA can regulate or depress a great amount of mRNAs, because miRNAs match the mRNAs only by the seed sequence which is comprised by the nucleotides from 2 to 7 or 8 [5]. The process of RNA silencing or RNA interference is completed of miRNAs, whose abnormal expression may give rise to many tumors, such as breast cancer, non-smallcell lung cancer and bladder cancer [1,6-8]. Recently, the personalized treatment of cancer patients has been developing with the deep study of molecular characteriza- 
tion of primary tumors [9]. Therefore, the integrated analysis of mRNA-miRNA is one of the focuses of the cancer studies.

In this study, we performed an integrative analysis of miRNA-mRNA based on aberrantly expressed miRNA and mRNA profiles in tumor cells by high-throughput sequencing and microarray techniques, respectively. According to their functional relationships and expression patterns, potential miRNA-mRNA interaction and regulatory network were comprehensively analyzed.

\section{Material and Methods}

HepG2 and L02 cells were obtained from American Type Tissue Collection and further sequenced. According to miRNA and mRNA expression profiles, differentially expressed miRNA and mRNA profiles were calculated between the two samples through fold change filtering. To acquire these mRNA/miRNA species, fold change values were assessed from normalized datasets which were the data from L02 cells. In this study, the cut-off values were 2 and 0.5 , which is utilized to identify whether the up-regulated and down-regulated mRNA/ miRNA species. The study was based on the fold change mRNA/miRNA to detect the aberrant expression between two kinds of cell.

The relationship between targets of miRNA from the miRTarBase database, the TargetScan software and the experimental results of this study were studied. Firstly, we utilized the miRNAs which had fold change in this work to predict the regulated mRNAs through the miRTarBase database which includes the validated miRNA targets. And through the TargetScan software, the targets of the same abnormal expression miRNAs were predicted. Then the accumulating frequency diagram and venn diagram directly presented the relationship of the data from the three groups. Finally, we compared the accuracy rate of the two methods.

Further, the functions of the predicted mRNAs were analyzed. Firstly, those mRNAs that were regulated by more than 2 differentially expressed miRNAs were selected, if their frequencies were more than 3. Functional enrichment analysis was used the CapitalBio MAS 3.0 software based on those aberrantly expressed mRNAs and predicted target mRNAs of abnormal miRNAs.

Those abundantly expressed miRNA gene families were selected from the abnormal miRNA expression profiles. miRNA members in the gene family have similar sequences, and may co-regulate biological processes. Therefore, we also analyzed their potential targets, especially some common targets. Simultaneously, further expression analysis was performed based on aberrantly expressed miRNA and mRNAs profiles.

\section{Results and Discussion}

\subsection{Similar Distribution Patterns across Different Target mRNA Datasets}

Herein, three methods were used to analyze targets of aberrantly expressed miRNAs (experimentally validated targets, predicted targets, and obtained abnormal mRNAs in the study). The accumulation frequency diagrams suggested diversity of targets based on dysregulated miRNAs. Some mRNAs were regulated by specific miRNAs, but others might be regulated by 2 or more different miRNAs (Figure 1). However, the frequency of down-regulated miRNAs is higher than up-regulated species. The venn diagram based on the miRTarBase database detected that the intersection of targets between up-regulated and down-regulated was 114 (32.02\% from up-regulated miRNAs, $17.54 \%$ from down-regulated species). The results from TargetScan program indicated that the intersection of targets between up-regulated and down-regulated is 3143 (82.17\% from up-regulated miRNAs, $54.44 \%$ from down-regulated species). Based

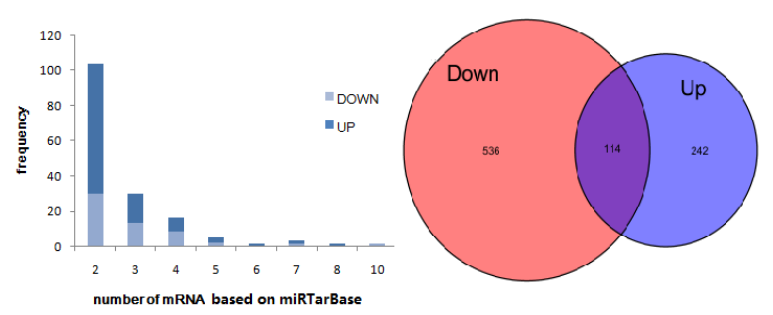

(a)

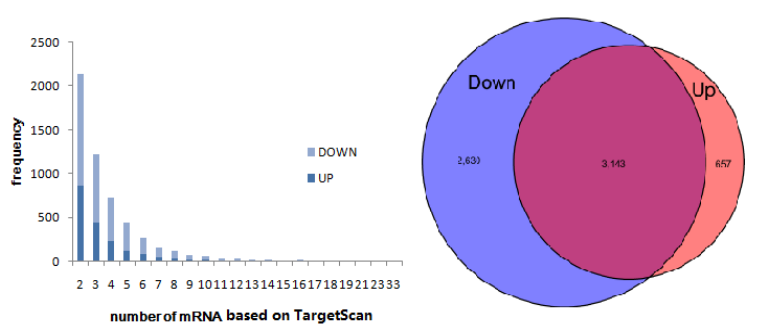

(b)

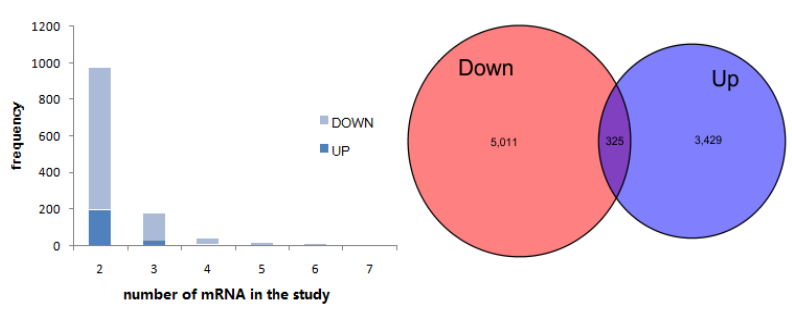

(c)

Figure 1. The distributions of the number of targets and the venn diagram of the aberrantly expressed miRNA profile. (a) is based on the data from the miRTarBase database; (b) is based on the data from the TargetScan program; (c) is based on the abnormal mRNA expression profile from the study. 
on special mRNA expression profiles in tumor cells, the study demonstrated that the intersection of up-regulated and down-regulated is 325 (8.66\% from up-regulated mRNA, 6.09\% from down-regulated mRNA).

These findings testified that each miRNA can regulate a series of targets; simultaneously each mRNA can be negatively regulated by several miRNAs (Figure 1). The reason was mainly derived from the flexible interaction between miRNA and mRNA through "seed sequences" and UTR. Moreover, we compared the accuracy rates of the two methods in predicting the target gene through chi-square test. No significant difference was detected between the two methods in predicting the targets, including targets of up-regulated and down-regulated miRNAs. Specially, in predicting the down-regulated mRNAs, the accuracy rates of the two methods are $29.84 \%$ and $28.03 \%\left(\chi^{2}=0.95, P=0.33\right)$, while in predicting the up-regulated targets, the accuracy rates of the two methods are $21.91 \%$ and $21.05 \%\left(\chi^{2}=0.14, P=0.70\right)$.

\subsection{Functional Enrichment Analysis Reveals Potential Contributions in Tumorigenesis}

More bioinformatics information about the different expression mRNAs and targets of abnormal miRNAs can be acquired from MAS 3.0, such as the pathways, the regulation networks and proteins. Herein, in order to further study their potential roles in occurrence and development of tumor, we also chose the pathway information from the KEGG (Kyoto Encyclopedia of Genes and Genomes) database [10]. One pathway was involved in a great amount of mRNAs. Simultaneously a specific mRNA also participated many pathways (Table 1). According to targets of deregulated miRNAs, and aberrantly expressed mRNA profiles in the study, important and essential biological processes could be enriched, including some human diseases. The pathways included MAPK signaling pathway, Wnt signaling pathway, Chronic myeloid leukemia, and etc. (Table 1). Further, we detected that the intersection of these three situations is MAPK signaling pathway, regulation of actin cytoskeleton and focal adhesion. Therefore, we conjectured that these pathways might trigger development or generation of each tumor or special liver cancer. The functions of key regulatory proteins of the actin cytoskeleton is regulating cancer cell migration and invasion though formation of invasive protrusions used by tumor cells, such as lamellipodia and invadopodia [11]. Wnt signaling pathway makes the hepatocelluar carcinoma dysregulated by two distinctive classes (CTNNB1 and Wnt-TGF $\beta$ ) [12].

\subsection{The Co-Rgulation of Homologous miRNAs in Gene Family}

We further selected a pair of miRNA family from the
Table 1. Pathways regulated by the different expression mRNAs.

\begin{tabular}{clcc}
\hline No. & Pathway & Count & $\boldsymbol{P}$-value \\
\hline 1 & MAPK signaling pathway & 59 & $2.15 \mathrm{E}-36$ \\
2 & Regulation of actin cytoskeleton & 54 & $7.13 \mathrm{E}-37$ \\
3 & Focal adhesion & 47 & $1.27 \mathrm{E}-30$ \\
4 & Wnt signaling pathway & 45 & $1.13 \mathrm{E}-34$ \\
5 & Axon guidance & 38 & $2.94 \mathrm{E}-29$ \\
6 & Focal adhesion & 42 & $4.35 \mathrm{E}-30$ \\
7 & Regulation of actin cytoskeleton & 31 & $1.02 \mathrm{E}-17$ \\
8 & MAPK signaling pathway & 30 & $4.98 \mathrm{E}-14$ \\
9 & Insulin signaling pathway & 26 & $3.49 \mathrm{E}-18$ \\
10 & Axon guidance & 24 & $1.11 \mathrm{E}-16$ \\
11 & MAPK signaling pathway & 27 & $3.63 \mathrm{E}-18$ \\
12 & Focal adhesion & 22 & $7.35 \mathrm{E}-16$ \\
13 & Regulation of actin cytoskeleton & 20 & $3.04 \mathrm{E}-13$ \\
14 & Wnt signaling pathway & 18 & $5.71 \mathrm{E}-14$ \\
15 & Chronic myeloid leukemia & 17 & $3.37 \mathrm{E}-18$ \\
\hline
\end{tabular}

*The 1 - 5 pathways of target mRNAs from up-regulated miRNAs; The 6 10 pathways of target mRNAs from down-regulated miRNAs; The 11 - 15 pathways are predicted by the intersection of them.

differentially expressed miRNA profiles. From the upregulation miRNAs, we chose the mir-8 gene family, including hsa-miR-200b-3p and hsa-miR-200a-3p (fold change value: 377.30 and 130.43) which had the maximum fold change value in from the mir-8 gene family (miR-200a, miR-200b, miR-200c, miR-141 and miR429). Secondly, we consulted the miRBase database to find the target of the two miRNAs. Obviously, they possessed some common target genes, including $B A P 1, S I P 1$, $W A S F 3, Z E B 1, Z E B 2$ and $Z F P M 2$, but they also regulated the different genes, respectively. Thirdly, we found the down-regulated mRNAs from the experimental data to check the predicted target genes. Finally, we utilized exact probability method to compare the accuracy rates between the co-regulated mRNAs and the other mRNAs. The intersection of the two miRNAs was 6 (46.15\% of the mRNAs regulated by hsa-mir-200b-3p, 33.33\% of the mRNAs regulated by hsa-mir-200a-3p) (Figure 2). The miRNA members in gene family shared the same or similar "seed sequences" and always showed consistent expression patterns, and they co-regulated multiple and essential biological processes. Therefore, we should pay attention to the miRNA family when we study miRNAs or the regulated mRNAs.

In conclusion, miRNA-mRNA interaction is much more complex than we thought. A specific miRNA may regulate multiple target mRNAs, and vice versa. The miRNA members in miRNA gene family may regulate the same targets and show consistent expression pat- 


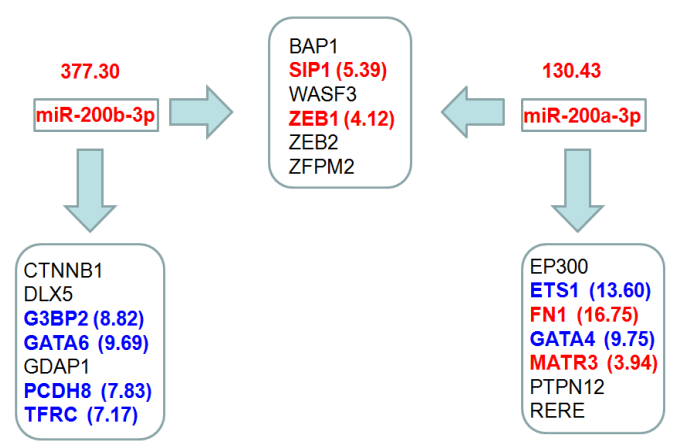

Figure 2. The experimentally validated targte mRNAs of hsa-miR-200a-3p and hsa-miR-200b-3p (members in mir-8 gene family). mRNAs or miRNAs in red are up-regulated in tumor cells; mRNAs in blue are down-regulated; and mRNAs in black stably express between normal cells. Furthermore, deregulated miRNAs or mRNAs are also highlighted fold change values.

terns in special time and space in vivo. The co-regulation processes will contribute to the robust network with multiple different molecules. Systematic analysis across different regulatory molecules should be crucial to study tumorigenesis at the multiple molecular levels, including mRNAs, miRNAs, lncRNAs, and etc.

\section{REFERENCES}

[1] E. Enerly, I. Steinfeld, K. Kleivi, S. K. Leivonen, M. R. Aure, H. G. Russnes, J. A. Ronneberg, H. Johnsen, R. Navon, E. Rodland, R. Makela, B. Naume, M. Perala, O. Kallioniemi, V. N. Kristensen, Z. Yakhini and A. L. Borresen-Dale, "miRNA-mRNA Integrated Analysis Reveals Roles for miRNAs in Primary Breast Tumors,” PLoS One, Vol. 6, 2011, Article ID: e16915.

http://dx.doi.org/10.1371/journal.pone.0016915

[2] F. Xie, P. Xiao, D. Chen, L. Xu and B. Zhang, "miRDeep Finder: A miRNA Analysis Tool for Deep Sequencing of Plant Small RNAs,” Plant Molecular Biology, 2012.

[3] D. P. Bartel, "MicroRNAs: Target Recognition and Regulatory Functions,” Cell, Vol. 136, 2009, pp. 215-233. http://dx.doi.org/10.1016/j.cell.2009.01.002

[4] C. Vaz, H. M. Ahmad, P. Sharma, R. Gupta, L. Kumar, R. Kulshreshtha and A. Bhattacharya, "Analysis of microRNA Transcriptome by Deep Sequencing of Small RNA Libraries of Peripheral Blood,” BMC Genomics, Vol. 11, 2010, p. 288. http://dx.doi.org/10.1186/1471-2164-11-288

[5] J. A. Broderick, W. E. Salomon, S. P. Ryder, N. Aronin and P. D. Zamore, "Argonaute Protein Identity and Pairing Geometry Determine Cooperativity in Mammalian RNA Silencing," RNA-A Publication of the RNA Society, Vol. 17, 2011, pp. 1858-1869. http://dx.doi.org/10.1261/rna.2778911
[6] F. M. Buffa, C. Camps, L. Winchester, C. E. Snell, H. E. Gee, H. Sheldon, M. Taylor, A. L. Harris and J. Ragoussis, "microRNA-Associated Progression Pathways and Potential Therapeutic Targets Identified by Integrated mRNA and microRNA Expression Profiling in Breast Cancer,” Cancer Research, Vol. 71, 2011, pp. 5635-5645. http://dx.doi.org/10.1158/0008-5472.CAN-11-0489

[7] R. Rosell, M. A. Molina, C. Costa, S. Simonetti, A. Gimenez-Capitan, J. Bertran-Alamillo, C. Mayo, T. Moran, P. Mendez, F. Cardenal, D. Isla, M. Provencio, M. Cobo, A. Insa, R. Garcia-Campelo, N. Reguart, M. Majem, S. Viteri, E. Carcereny, R. Porta, B. Massuti, C. Queralt, I. de Aguirre, J. M. Sanchez, M. Sanchez-Ronco, J. L. Mate, A. Ariza, S. Benlloch, J. J. Sanchez, T. G. Bivona, C. L. Sawyers and M. Taron, "Pretreatment EGFR T790M Mutation and BRCA1 mRNA Expression in ErlotinibTreated Advanced Non-Small-Cell Lung Cancer Patients with EGFR Mutations,” Clinical Cancer Research, Vol. 17, 2011, pp. 1160-1168. http://dx.doi.org/10.1158/1078-0432.CCR-10-2158

[8] A. Font, M. Taron, J. L. Gago, C. Costa, J. J. Sanchez, C. Carrato, M. Mora, P. Celiz, L. Perez, D. Rodriguez, A. Gimenez-Capitan, V. Quiroga, S. Benlloch, L. Ibarz and R. Rosell, "BRCA1 mRNA Expression and Outcome to Neoadjuvant Cisplatin-Based Chemotherapy in Bladder Cancer,” Annals of Oncology, Vol. 22, 2011, pp. 139-144. http://dx.doi.org/10.1093/annonc/mdq333

[9] A. M. Sieuwerts, B. Mostert, J. Bolt-de Vries, D. Peeters, F. E. de Jongh, J. M. Stouthard, L. Y. Dirix, P. A. van Dam, A. Van Galen, V. de Weerd, J. Kraan, P. van der Spoel, R. Ramirez-Moreno, C. H. van Deurzen, M. Smid, J. X. Yu, J. Jiang, Y. Wang, J. W. Gratama, S. Sleijfer, J. A. Foekens and J. W. Martens, "mRNA and microRNA Expression Profiles in Circulating Tumor Cells and Primary Tumors of Metastatic Breast Cancer Patients,” Clinical Cancer Research, Vol. 17, 2011, pp. 3600-3618. http://dx.doi.org/10.1158/1078-0432.CCR-11-0255

[10] H. Ogata, S. Goto, K. Sato, W. Fujibuchi, H. Bono and M. Kanehisa, "KEGG: Kyoto Encyclopedia of Genes and Genomes,” Nucleic Acids Research, Vol. 27, 1999, pp. 29-34. http://dx.doi.org/10.1093/nar/27.1.29

[11] H. Yamaguchi and J. Condeelis, "Regulation of the Actin Cytoskeleton in Cancer Cell Migration and Invasion," Biochimica et Biophysica Acta, Vol. 1773, 2007, pp. 642652. http://dx.doi.org/10.1016/j.bbamcr.2006.07.001

[12] A. Lachenmayer, C. Alsinet, R. Savic, L. Cabellos, S. Toffanin, Y. Hoshida, A. Villanueva, B. Minguez, P. Newell, H. W. Tsai, J. Barretina, S. Thung, S. C. Ward, J. Bruix, V. Mazzaferro, M. Schwartz, S. L. Friedman and J. M. Llovet, "Wnt-Pathway Activation in Two Molecular Classes of Hepatocellular Carcinoma and Experimental Modulation by Sorafenib,” Clinical Cancer Research, Vol. 18, 2012, pp. 4997-5007. http://dx.doi.org/10.1158/1078-0432.CCR-11-2322 